\title{
Efficient near-ML Decoding via Statistical Pruning ${ }^{1}$
}

\author{
Radhika Gowaikar, Babak Hassibi \\ Dept. of Electrical Engineering, \\ California Institute of Technology, CA 91125 \\ \{govaikar, hassibi\}ecaltech.edu
}

\begin{abstract}
Maximum-likelihood (ML) decoding often reduces to finding the closest (skewed) lattice point in $N$-dimensions to a given point $x \in \mathcal{C}^{N}$. Sphere decoding is an algorithm that does this. We modify the sphere decoder to reduce the computational complexity of decoding while maintaining near-ML performance.
\end{abstract}

\section{System Model}

We assume a discrete-time, block-fading, multiple-antenna channel model with $N$ transmit and $N$ receive antennas, where the channel is known to the receiver. If $\mathcal{S}$ is the signal space, the transmitted signal $\tilde{s} \in \mathcal{S}^{N \times 1}$ and the received signal $x \in \mathcal{C}^{N \times 1}$ are related by $x=\sigma_{h} H \tilde{s}+v$ where $H \in \mathcal{C}^{N \times N}$ is the known channel matrix and $v \in \mathcal{C}^{N \times 1}$ is the additive noise vector. Both are comprised of i.i.d. complex-Gaussian entries $\mathcal{C N}(0,1) . \sigma_{h}$ determines the SNR. Under these conditions the ML criterion requires us to find $s \in \mathcal{S}^{N \times 1}$ that minimizes $\|x-H s\|^{2}$.

\section{Sphere Decoder}

The sphere decoder finds lattice points in a hypersphere of radius $r$ centered at $x$, i.e., it finds all $s \in \mathcal{S}^{N \times 1}$ that satisfy $r^{2} \geq\|x-H s\|^{2}$. For this, we decompose $H$ as $H=Q R$ where $Q$ is unitary and $R$ is upper triangular with positive diagonal. (Both are $N \times N$.) Then $\|x-H s\|^{2}=\left\|Q^{*} x-R s\right\|^{2}$. Define $y^{\prime}=Q^{*} x-R s$ and $\lambda_{i}=\left|y_{N-i+1}^{\prime}\right|^{2}$ for $i=1,2, \ldots, N$. We need to solve $\lambda_{1}+\lambda_{2}+\ldots+\lambda_{N} \leq r^{2}$. This is done by solving successively for $\lambda_{1} \leq r^{2} ; \lambda_{1}+\lambda_{2} \leq r^{2} ; \ldots ; \lambda_{1}+\lambda_{2}+\cdots+\lambda_{N} \leq r^{2}$. This can be done because the first condition gives an interval for $s_{N}$, whereas for any pre-determined $s_{N}, \ldots, s_{N-i+2}$, the $i$-th condition gives an interval for $s_{N-i+1}$. While the sphere decoder avoids exhaustive search it does incur very high computational complexities for very large $N[1]$. This happens because to have a high probability of finding at least one point in the hypersphere, $r$ has to be proportional to $N$ and a very large fraction of the points is retained in the early dimensions.

\section{Statistical Pruning}

To prune the search space right from the smaller dimensions, we modify the sphere decoder. We determine a schedule of radii $r_{1} \leq r_{2} \leq \ldots \leq r_{N}$ and solve for $\lambda_{1} \leq r_{1}^{2} ; \lambda_{1}+\lambda_{2} \leq$ $r_{2}^{2} ; \ldots ; \lambda_{1}+\lambda_{2}+\cdots+\lambda_{N} \leq r_{N}^{2}$. Call this region $\mathcal{D}$. Since $\mathcal{D}$ is not hyperspherical, this is not exact ML decoding. If $\epsilon$ is the probability that the transmitted vector is not in $\mathcal{D}$, a loose upper bound on the probability of error is $P_{e} \leq P_{e}^{M L}+\epsilon$. The quantity $\epsilon$ can be determined exactly in terms of the $r_{i}$ s and so the $r_{i}$ s can be chosen to make $\epsilon$ as small as desired to ensure near-ML performance.

\footnotetext{
${ }^{1}$ This work was supported in part by the National Science Foundation under grant no. CCR-0133818, by the office of Naval Research under grant no. N00014-02-1-0578, and by Caltech's Lee Center for Advanced Networking.
}

\section{RESULTS}

The expected computational complexity $C$ is given by $\sum_{k=1}^{N}$ (expected \# of points in $\left.\mathcal{D}_{k}\right) \cdot$ (flops/point) where $\mathcal{D}_{k}$ is the restriction of $\mathcal{D}$ to the $k$-th dimension. The expected \# of points in $\mathcal{D}_{k}$ is given by $\sum_{s^{k} \in \mathcal{S}^{k \times 1}} P\left(s^{k} \in \mathcal{D}_{k}\right)$ and flops $/$ point $=8 k+32$ for the $k$-th dimension. An upper bound on $P\left(s^{k} \in \mathcal{D}_{k}\right)$ leads to

$$
C \leq \sum_{k=1}^{N}(8 k+32) \sum_{l=0}^{2 k}\left(\begin{array}{c}
2 k \\
l
\end{array}\right) \Gamma\left(\frac{r_{k}^{2}}{1+\sigma_{h}^{2} l}, k\right)
$$

for QPSK constellations. Here $\Gamma(x, a)=\int_{0}^{x} \frac{e^{-t}}{\Gamma(a)} t^{a-1} d t$. An approximation to $P\left(s^{k} \in \mathcal{D}_{k}\right)$ leads to

$$
C \approx \sum_{k=1}^{N}(8 k+32) \sum_{s^{k} \in \mathcal{S}^{k} \times 1} \prod_{j=1}^{k} \min \left(1, \frac{X_{k}}{2\left(1+\sigma_{h}^{2}\left\|s^{j}-\tilde{s}^{j}\right\|^{2}\right.}\right)
$$

where the $X_{i}$ s depend on $s^{i}-\tilde{s}^{i}$ and can be obtained recursively. This is computed efficiently with Monte Carlo simulations.

\section{Simulations}

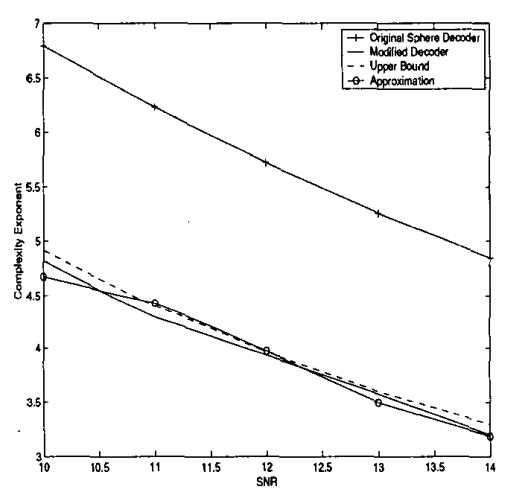

Figure 1: Complexity Exponent for $N=50$ with QPSK

Fig. (1) shows the complexity exponent $(\log C / \log N)$ for $N=50$ with the sphere decoder as well as the modified algorithm. The approximation and the upper bound are also plotted. A linear schedule of radii $r_{i}^{2}=(\delta \log N+i)$ with $\delta$ chosen to make $\epsilon=0.01$ was used. A computational savings of $50^{2}=2500$ is observed.

\section{Conclusions}

The algorithm reduces decoding complexity by exploiting the statistics of the problem. Performance can be made arbitrarily close to ML by choice of $\epsilon$.

\section{REFERENCES}

[1] B. Hassibi and H. Vikalo, "The complexity of sphere decoding. pt. I Expected Complexity ," Submitted to IEEE Trans. Sig. Proc.; 2003 